Original Research Paper

\title{
Some Contributions to the Theory of Abstract Degenerate Volterra Integro-Differential Equations
}

\author{
Marko Kostić \\ Faculty of Technical Sciences, University of Novi Sad, Trg D. Obradovica 6, 21125 Novi Sad, Serbia
}

Article history

Received: 24-03-2016

Revised: $15-06-2016$

Accepted: 17-06-2016

Email: marco.s@verat.net

\begin{abstract}
In this study, we contribute to the existing theory of abstract degenerate Volterra integro-differential equations in sequentially complete locally convex spaces. We investigate a class of abstract degenerate Volterra inclusions by using the multivalued linear operator approach, as well as a class of abstract degenerate multi-term fractional differential equations with Caputo derivatives by using the pure Laplace transform techniques.
\end{abstract}

Keywords: Abstract Degenerate Volterra Inclusions, Multivalued Linear Operators, Multi-Term Degenerate Fractional Differential Equations, Caputo Derivatives, Locally Convex Spaces

Mathematics Subject Classification, 47D06, 47D60, 47D62, 47D99

\section{Introduction and Preliminaries}

As mentioned in the abstract, the main aim of this paper is to contribute to the existing theory of abstract degenerate Volterra integro-differential equations in sequentially complete locally convex spaces (for the basic source of information on the abstract degenerate differential equations with integer order derivatives, we refer the reader to the monographs (Carroll and Showalter, 1976; Demidenko and Uspenskii, 2003; Favini and Yagi, 1998; Melnikova and Filinkov, 2001; Sviridyuk and Fedorov, 2003). The study of abstract degenerate Volterra equations starts presumably with the paper by Favini and Tanabe (2001), while the study of abstract degenerate fractional differential equations starts presumably with the paper by Fedorov and Debbouche (2013). In this study, we continue our research studies (Kostić, 2015b; 2016; 0000c) by investigating the abstract degenerate multi-term Volterra inclusions and the abstract degenerate multi-term fractional differential equations with Caputo derivatives (cf. (Kim, 2002; Knuckles and Neubrander, 1994; Obukhovskii and Zecca, 2003), the forthcoming monograph (Kostić, 0000a) and references cited therein for further information on the above subjects).

The organization of paper can be briey described as follows. In section 2, we repeat some known facts and definitions about multivalued linear operators in locally convex spaces. The main purpose of section 3 is to prove some results on the well-posedness of the following abstract degenerate multi-term Volterra inclusion:

$$
0 \in B u(t)+\sum_{j=0}^{n-1} A_{j}\left(a_{j}^{*} u\right)(t)+F(t), t \in[0, \tau)
$$

where, $n \in N, 0<\tau \leq \infty, F:[0, \tau) \rightarrow P(Y), a_{0}, \cdots, a_{n-1}$ $\in L_{l o c}^{1}([0, \tau))$ and $A \equiv A_{0}, \cdots, A_{n-1}, B \equiv A_{n}$ are multivalued linear operators acting between the sequentially complete locally convex spaces $\mathrm{X}$ and $\mathrm{Y}$ (for some special subcases of problem (1.1), cf. [(Kostić, 2015b), (3.7)] and [(Kostić, 0000b), (2.1)]). In Definition 3.1, we introduce the notion of a $\mathrm{V}$-(pre)-solution of problem (1.1), while in Theorem 3.4 and Theorem 3.5 we analyze subordination principles for $V$-solutions of problem (1.1). The classes of (pre)- $\left(k, C_{1}, V\right)$-existence families for (1.1) and $\mathrm{k}$-regularized $\mathrm{C}_{2}$-uniqueness families for (1.1) are introduced in Definition 3.6 and Definition 3.7 (here, $V \subseteq N_{n}^{0}$ ). The main objective of Proposition 3.8 and Theorem 3.9-Theorem 3.12 is to extend some known theoretical results from [(Kostić, 2015b), section 3] to the introduced classes of existence and uniqueness families (because of a great similarity with our previous research study (Kostić, 2015b), the proof is given only for Theorem 3.11). Without going into exceptional details, we will demonstrate how our results can be used to provide certain applications at the end of section 3 .

In section 4, we continue our previous research of entire solutions of higher order degenerate Cauchy problems with integer order derivatives (Kostić, 2016) by investigating (cf. Theorem 4.2) the existence and uniqueness of analytical solutions of abstract degenerate multi-term Cauchy problem: 


$$
V \subseteq N_{n}^{0}
$$

where, $n \in N \mid\{1\}, A, B$ and $\mathrm{A}_{1}, \cdots A_{n-1}$ are closed linear operators on a sequentially complete locally convex space $X, 0 \leq \alpha_{1}<\cdots<\alpha_{n}, 0 \leq \alpha<\alpha_{n}, f(t)$ is a continuous $\mathrm{X}$ valued function and $D_{t}^{\alpha}$ denotes the Caputo fractional derivative of order $\alpha$ (Kim, 2002; Kostić, 2015a)). We apply Theorem 4.2 in the analysis of fractional analogons of abstract Boussinesq-Love equation, for which it is known that plays an important role in the modeling the longitudinal waves in an elastic bar with the transverse inertia and the fractional analogons of abstract Barenblatt-Zheltov-Kochina equation, for which it is known that plays an important role in the study of fluid filtration in fissured rocks (cf. Example 4.4).

We use the standard notation throughout the paper. Unless specified otherwise, we assume that $X$ is a Hausdorff sequentially complete locally convex space over the field of complex numbers, SCLCS for short. If $Y$ is also an SCLCS over the field of complex numbers, then we denote by $L(Y, X)$ the space consisting of all continuous linear mappings from $Y$ into $X ; L(X) \equiv L(X$, $X)$. By $\otimes_{X}(\otimes$, if there is no risk for confusion), we denote the fundamental system of seminorms which defines the topology of $X$. The fundamental system of seminorms which defines the topology on $Y$ is denoted by $\otimes_{\mathrm{Y}}$. The symbol $I$ denotes the identity operator on $X$. Assume $0<\tau \leq \infty$. Then a strongly continuous operator family $(W(t))_{t \in[0, \tau)} \subseteq L(Y, X)$ is said to be locally equicontinuous iff, for every $T \in(0, \tau)$ and for every $p \in \otimes_{X}$, there exist $q_{p} \in \otimes_{Y}$ and $c_{p}>0$ such that $p(W(t) y) \leq c_{p} q_{p}(y), \quad y \in Y, \quad t \in[0, \quad T] ;$ the notions of equicontinuity of $(W(t))_{t \in[0, \tau)}$ and the exponential equicontinuity of $(W(t)) t \geq 0$ are defined similarly. Suppose that $A$ is a closed linear operator acting on $X$. Then we denote the domain, kernel space and range of $A$ by $D(A), N(A)$ and $R(A)$, respectively. Since no confusion seems likely, we will identify $A$ with its graph. Set $p_{A}(x):=p(x)+p(A x), x \in D(A), p \in \otimes$. Then the calibration $\left(p_{A}\right)_{p \in \otimes}$ induces the Hausdorff sequentially complete locally convex topology on $D(A)$; we denote this space simply by $[D(A)]$.

If $V$ is a general topological vector space, then a function $f: \Omega \rightarrow V$, where is an open non-empty subset of $C$, is said to be analytic if it is locally expressible in a neighborhood of any point $z \in \Omega$ by a uniformly convergent power series with coefficients in $V$. We refer the reader to [(Arendt et al., 2001; Kostić, 2015a), section 1.1] and references cited there for the basic information about vector-valued analytic functions. In our approach the space $X$ is sequentially complete, so that the analyticity of a mapping $f: \Omega \rightarrow X$ is equivalent with its weak analyticity. It is said that a function $\mathrm{f}$ : [0, $\infty) \rightarrow E$ is locally Holder continuous with the exponent $r \in(0,1]$ iff for each $p \in \otimes$ and $T>0$ there exists $\mathrm{M} \geq 1$ such that $p(f(t)-f(s)) \leq M|\mathrm{t}-\mathrm{s}|^{\mathrm{r}}$, provided $0 \leq t, s \leq T$. The reader may consult [(Prüss, 1993), Definition 4.5, p. 96] for the notion of a completely positive function on $[0, \infty)$ (cf. also [(Lizama, 2000), Remark 3.6, (3.3)]). Concerning the integration of functions with values in sequentially complete locally convex spaces, we will follow the approach of cf. [(Martinez and Sanz, 2001), pp. 99-102]).

Sometimes we use the following condition on a scalar-valued function $K(\cdot):(P 1) \quad K(\cdot)$ is Laplace transformable, i.e., it is locally integrable on $[0, \infty)$ and there exists $\beta \in R$ so that:

$$
\tilde{K}(\lambda):=L(K)(\lambda):=\lim _{b \rightarrow \infty} \int_{0}^{b} e^{-\lambda t} K(t) d t:=\int_{0}^{\infty} e^{-\lambda t} K(t) d t
$$

Exists for all $\lambda \in C$ with $\operatorname{Re} \lambda>\beta$. Put $\operatorname{abs}(K):=\inf \{\operatorname{Re}$ $\lambda: \tilde{K}(\lambda)$ existsg $\}$ and denote by $L^{-1}$ the inverse Laplace transform.

We say that a function $h(\cdot)$ belongs to the class $L T-X$ iff there exists a function $f \in C([0, \infty): X)$ such that for each $p \in \otimes$ there exists $M_{p}>0$ satisfying $p(f(t)) \leq M_{p} e^{a t}$, $t \geq 0$ and $h(\lambda)=(L f)(\lambda), \lambda>a$. The set consisting of all originals $f(t)$ will be denoted by $L T_{o r}-X$. In (Kostić, $0000 \mathrm{c}$ ), we have recently proposed a new theoretical method for concept useful in the analysis of operational properties of Laplace transform of non-continuous functions with values in sequentially complete locally convex spaces. If $f:[0, \infty) \rightarrow X$ is a locally Lebesgue integrable function (in the sense of [(Kostić, 2015a) Definition 1.1.5(i)], with $\mu$ being the Lebesgue measure) and $\tilde{f}\left(\lambda_{0}\right)$ exists for some $\lambda_{0} \in C$, then we define the abscissa of convergenece of $\tilde{f}(\cdot)$ by:

$$
\operatorname{abs}(f):=\inf \{\Re \lambda: \tilde{f}(\lambda) \text { exists }\}
$$

Otherwise, $a b s(f):=+\infty$. It is said that $f(\cdot)$ is Laplace transformable, or equivalently, that $f(\cdot)$ belongs to the class $(P 1)-X$, iff $\operatorname{abs}(f)<\infty$. The reader may consult [(Arendt et al., 2001; Xiao and Liang, 1998), Chapter 1], [(Kostić, 2015a), Section 1.2] and (Kostić, 0000c) for the basic properties of vector-valued Laplace transform.

Given $\theta \in(0, \pi]$ in advance, define $\Sigma_{\theta}:=\{\lambda \in C\}: \lambda \neq 0$, $|\arg (\lambda)|<\theta\}$. Further on, $\lceil\beta\rceil:=\inf \{n \in Z: \beta \leq n\}(\beta \in R)$. A scalar-valued function $k \in L_{\text {loc }}^{1}[(0, \tau))$ is said to be a kernel on $[0, \tau)$ iff for any scalar-valued continuous function $t \mapsto u(t), \quad t \in[0, \tau)$, the preassumption $\int_{0}^{t} k(t-s) u(s) d s=0, t \in[0, \tau)$ implies $u(t)=0, t \in[0, \tau)$. The Gamma function is denoted by $\Gamma(\cdot)$ and the principal branch is always used to take the powers; the convolution like mapping $*$ is given 
by $f^{*} g(t):=\int_{0}^{t} f(t-s) g(s) d s$. Set $g_{\zeta}(t):=\mathrm{t}^{\zeta-1} / \Gamma(\zeta), 0^{\zeta}:=$ $0(\zeta>0, t>0)$ and $g_{0}(t):=$ the Dirac $\delta$-distribution. If $f$ : $[0, \infty) \rightarrow X$ is a continuous function, then we set $g_{0} * f \equiv f$.

During the past three decades, considerable interest in fractional calculus and fractional differential equations has been stimulated due to their numerous applications in various fields of science and engineering. Basic information about fractional calculus and non-degenerate fractional differential equations can be obtained by consulting (Bazhlekova, 2001; Diethelm, 2010; Kilbas et al., 2006; Kostić, 2015a; Podlubny, 1999; Prüss, 1993; Samko et al., 1993) and the references cited therein.

In this study, we will use the Caputo fractional derivatives. Let $\zeta>0$. Then the Caputo fractional derivative $D_{\zeta}^{t} u$ (Bazhlekova, 2001; Kostić, 2015a) is defined for those functions $u \in C^{\zeta \zeta-1}([0, \infty): X)$ for which

$$
\begin{gathered}
g_{\lceil\zeta\rceil-\zeta} *\left(u-\sum_{j=0}^{\lceil\zeta\rceil-1} u^{(j)}(0)_{g j+1}\right) \in C^{\lceil\zeta\rceil}([0, \infty): X), \text { by: } \\
D_{t}^{\zeta} u(t):=\frac{d^{\lceil\zeta\rceil}}{d t^{\lceil\zeta\rceil}}\left[g\lceil\zeta\rceil-\zeta *\left(u-\sum_{j=0}^{\lceil\zeta\rceil-1} u^{(j)}(0)_{g j+1}\right)\right]
\end{gathered}
$$

If the Caputo fractional derivative $D_{t}^{\zeta} u(t)$ exists, then for each number $v \in(0, \zeta)$ the Caputo fractional derivative $D_{t}^{v} u(t)$ exists, as well.

The Mittag-Leffler function $E_{\beta, \gamma}(z)(\beta>0, \gamma \in R)$ is defined by:

$$
E_{\beta, \gamma}(z):=\sum_{k=0}^{\infty} \frac{z^{k}}{\Gamma(\beta k+\gamma)}, z \in C
$$

In this place, we assume that $1 / \Gamma(\beta k=\gamma)=0$ if $\beta k+$ $\gamma \in-N_{0}$. Set, for short, $E_{\beta}(z):=E_{\beta, 1}(z), z \in C$. Let $\beta \in(0,1)$. Then the Wright function $\Phi_{\beta}(\cdot)$ is defined by:

$$
\Phi \beta(t):=L^{-1}\left(E_{\beta}(-\lambda)\right)(t), t \geq 0
$$

For further information about the Mittag-Leffler and Wright functions, cf. (Bazhlekova, 2001; Kostić, 2015a) and the references cited there.

Let $0<\tau \leq \infty$ and $F:[0, \tau) \rightarrow P(X)$ : A single-valued function $f:[0, \tau) \rightarrow X$ is called a section of $F$ iff $f(t) \in F(t)$ for all $t \in[0, \tau)$. We denote the set consisting of all continuous sections of $F$ by $\sec _{c}(F)$.

\section{Multivalued Linear Operators in Locally Convex Spaces}

In this section, we will present some necessary definitions from the theory of multivalued linear operators. For more details about this topic, we refer the reader to the monographs by Cross (1998) and Favini and Yagi (1998).

Let $X$ and $Y$ be two sequentially complete locally convex spaces over the field of complex numbers. A multivalued map $A: X \rightarrow P(Y)$ is said to be a Multivalued Linear Operator (MLO) iff the following holds:

- $D(A):=\{x \in X: A x \neq \lambda \phi\}$ is a linear subspace of $X$

- $A x+A y \subseteq A(x+y), x, y \in D(A)$ and $\lambda A x \subseteq A(\lambda x)$, $\lambda \in C, x \in D(A)$

If $X=Y$, then we say that $A$ is an MLO in $X$. As an almost immediate consequence of definition, we have that the equality $\lambda A x+\eta A y=A(\lambda x+\eta y)$ holds for every $x, y \in D(A)$ and for every $\lambda, \eta \in C$ with $|\lambda|+|\eta| \neq 0$. If $A$ is an MLO, then $A 0$ is a linear manifold in $Y$ and $A x=f+$ $A 0$ for any $x \in D(A)$ and $f \in A x$. Define $R(A):=\{A x$ : $x \in D(A)\}$. Then the set $N(A):=A^{-1} 0=\{x \in D(A): 0 \in A x\}$ is called the kernel of $A$. The inverse $A^{-1}$ of an MLO is defined by $D\left(A^{-1}\right):=R(A)$ and $A^{-1} y:=\{x \in D(A)$ : $y \in A x\}$. It can be easily seen that $A^{-1}$ is an MLO in $X$, as well as that $N\left(A^{-1}\right)=A 0$ and $\left(A^{-1}\right)^{-1}=A$. If $N(A)=\{0\}$, i.e., if $A^{-1}$ is single-valued, then $\mathrm{A}$ is said to be injective. If $A, B: X \rightarrow P(Y)$ are two MLOs, then we define its sum $A+B$ by $D(A+B):=D(A) \cap D(B)$ and $(A$ $+B) x:=A x+B x, x \in D(A+B)$, It is clear that $A+B$ is likewise an MLO. We write $A \subseteq B$ iff $D(A) \subseteq D(B)$ and $A x \subseteq B x$ for all $x \in D(A)$.

Let $A: X \rightarrow P(Y)$ and $B: Y \rightarrow P(Z)$ be two MLOs, where $Z$ is an SCLCS. The product of $A$ and $B$ is defined by $D(B A):=\{x \in D(A): D(B) \cap A x \neq \phi\}$ and $B A x$ $:=B(D(B) \cap A x)$. Then $B A: X \rightarrow P(Z)$ is an MLO and $(B A)^{-1}=A^{-1} B^{-1}$. The scalar multiplication of an MLO $A: X \rightarrow P(Y)$ with the number $z \in C, z A$ for short, is defined by $D(z A):=D(A)$ and $(z A)(x):=z A x, x \in D(A)$ : It is clear that $z A: X \rightarrow P(Y)$ is an MLO and $(\omega z) A=$ $\omega(z A)=z(\omega A), z, \omega \in C$.

We say that an MLO $A: X \rightarrow P(Y)$ is closed if for any nets $\left(x_{\tau}\right)$ in $D(A)$ and $\left(y_{\tau}\right)$ in $Y$ such that $y_{\tau} \in A x_{\tau}$ for all $\tau \in I$ we have that the suppositions $\lim _{\tau \rightarrow \infty} x_{\tau}=x$ and $\lim _{\tau \rightarrow \infty} y_{\tau}=y$ imply $x \in D(A)$ and $y \in A x$. As mentioned below, some of our results hold even for relatively closed MLOs (Knuckles and Neubrander, 1994).

We need the following auxiliary lemma from (Kostić, 0000c).

\section{Lemma 2.1}

Let $\Omega$ be a locally compact, separable metric space and let $\mu$ be a locally finite Borel measure defined on $\Omega$. Suppose that $A: X \rightarrow P(Y)$ is a closed MLO. Let $f: \Omega \rightarrow X$ and $g: \Omega \rightarrow Y$ be $\mu$-integrable and let $g(x) \in A f(x), x \in \Omega$. Then $\int_{\Omega} d \mu \in D(A)$ and $\int_{\Omega} d \mu \in A \int_{\Omega} f d \mu$. 
In the remaining part of paper, $\Omega$ will always be an appropriate subspace of $R$ and $\mu$ will always be the Lebesgue measure defined on $\Omega$.

We need also the following lemma from (Kostić, 0000c), which can be proved by using the PhragmenDoetsch inversion formula for the Laplace transform.

\section{Lemma 2.2}

Suppose that $A: X \rightarrow P(Y)$ is a closed MLO, as well as that $f \in(P 1)-X, l \in(P 1)-Y$ and $(\tilde{f}(\lambda), \tilde{l}(\lambda)) \in A, \lambda \in C$ for $\mathfrak{R} \lambda>\max (\operatorname{abs}(f), a b s(l))$. Then $l(t) \in A f(t)$ for any $\mathrm{t} \geq 0$ which is a point of continuity of both functions $f(t)$ and $l(t)$.

\section{On a Class of Abstract Degenerate Volterra Inclusions in Locally Convex Spaces}

In this section, we will consider the degenerate Volterra integral inclusion (1.1) in more detail. Let us recall that $n \in N, 0<\tau \leq \infty, F:[0, \tau) \rightarrow P(Y), a_{0}, \cdots, a_{n-1} \in$ $L^{1}{ }_{l o c}([0, \tau))$, as well as that $A \equiv A_{0}, \cdots, A_{n-1}, B \equiv A_{n}$ are multivalued linear operators acting between the sequentially complete locally convex spaces $X$ and $Y$. Set $a_{n}(t):=\delta$-distribution and $\tilde{\delta}:=1$.

In the following definition, we introduce the notion of a $V$-(pre)-solution of problem (1.1).

\section{Definition 3.1. Suppose $V \subseteq N_{n}^{0}$}

A function $\mu \in C([0, \tau): X)$ is said to be a $V$-presolution of problem (1.1) iff there exist functions $\mu_{j}$ $\in C([0, \tau): Y) \quad\left(j \in N_{n}^{0} \backslash V\right)$ such that $u_{j}(t) \in A_{j} u(t), t \in[0$, $\tau), j \in N_{n}^{0} \backslash V,\left(a_{j}^{*} u\right)(t) \in D\left(A_{j}\right), t \in[0, \tau), j \in V$ and:

$$
0 \in \sum_{j \in N_{n}^{0}}\left(a_{j} * u_{j}\right)(t)+\sum_{j \in v} A_{j}\left(a_{j} * u\right)(t)+F(t), t \in[0, \tau)
$$

A function $u \in C([0, \tau): X)$ is said to be a $\mathrm{V}$-solution of problem (1.1) iff there exist functions $u_{j} \in C([0, \tau): Y)$ $\left(j \in N_{n}^{0} \backslash V\right)$ and $u_{j}, A_{j} \in C([0, \tau): Y) \quad(j \in V)$ such that $u_{j}(t) \in A_{j} u(t), t \in[0, \tau), \quad j \in N_{n}^{0} \backslash V, u_{j}, A_{j}(t) \in A_{j}\left(a_{j}^{*} u\right)(t)$, $t \in[0, \tau), j \in V$ and:

$$
0 \in \sum_{j \in N_{n}^{0} \backslash V}\left(a_{j} * u_{j}\right)(t)+\sum_{j \in V} u_{j}, A_{j}(t)+F(t), t \in[0, \tau)
$$

Suppose that $V \subseteq N_{n}^{0}$ and $u(t)$ is a $V$-solution of problem (1.1). Then it is clear that $\sec _{c}(F) \neq \phi$. Furthermore, if $V_{1} \subseteq V_{2} \subseteq N_{n}^{0}$, then we can simply prove with the help of Lemma 2.1 that any $V_{1}$-(pre)-solution of problem (1.1) is a $V_{2}$-(pre)-solution of (1.1), provided that the operators $A_{j}$ are closed for $j \in V_{2} \backslash V_{1}$. If $V=\phi$ then the notion of a pre-solution of (1.1) coincides with that of solution of (1.1); if this is the case, then any solution of (1.1) is also said to be a strong solution of (1.1). Here it is worth noting that the assumption $u \in C([0, \tau): X)$ in Definition 3.1 (i) is slightly redundant in the case that $V$ $=\phi$ becuse then we do not have a problem with defining the convolutions $\left(a_{j}^{*} u\right)(t)$ for $j \in V$. Assuming $V=\phi$ and all the remaining assumptions from Definition 3.1(i) hold, $u(t)$ will be called a p-strong solution of problem (1.1). In the case that $n=1$, the most important examples of p-strong solutions of problem (1.1) with $a_{0}(t)=g_{\alpha}(t)$, where $\alpha>0$, are obtained by integrating $\alpha$-times psolutions of abstract Degenerate Fractional Problem $(\mathrm{DFP})_{R}$; cf. [(Kostić, 0000c), Definition 4.1(ii)] for the notion and (Favini and Yagi, 1998) for a great number of important examples with $\alpha=1$. We refer the reader to [(Kostić, 2015b), Example 2.5] and [(Kostić, 0000c), Example 5.8] for some examples of (multi-term) degenerate Cauchy problems whose solutions can be defined only locally.

\section{Remark 3.2}

Suppose that $\mathrm{A}_{0}, \cdots, \mathrm{A}_{\mathrm{n}-1}, B$ are single-valued linear operators between the spaces $X$ and $Y$ and the mapping $f$ : $[0, \tau) \rightarrow D(B)$ is given in advance. Consider the following degenerate Volterra integral equation:

$$
0=B u(t) \sum_{j=0}^{n-1} A_{j}\left(a_{j}^{*} u\right)(t)+B f(t), t \in[0, \tau)
$$

which is, unquestionably, the most important sub case of (1.1). Along with this equation, we consider the accompanied problem of type (1.1):

$$
0 \in u(t) \sum_{j=0}^{n-1} B^{-1} A_{j}\left(a_{j}^{*} u\right)(t)+f(t), t \in[0, \tau)
$$

with the multivalued linear operators $A_{0}:=B^{-1} A_{0}, \cdots, A_{n-1}$ $:=B^{-1} A_{n-1} ; B:=I$ in $X$. Let $V \subseteq N_{n}^{0}$. Then the following holds:

- If $V=N_{n}^{0}$, then any $V$-pre-solution of problem (3.3) is a $V$-pre-solution of problem (3.2)

- If $B \in L(X, Y)$, then any $V$-(pre)-solution of problem (3.3) is a $V$-(pre)-solution of problem (3.2)

\section{Remark 3.3}

Suppose that $\tau=\infty,\left|a_{j}\right|(t)(0 \leq j \leq n-1)$ satisfy (P1) and the MLOs $A_{j}$ are closed for $0 \leq j \leq n$. Let $u \in C([0, \tau): X)$, $u_{j} \in C([0, \tau): Y) \quad j \in N_{n}^{0} \backslash V$ and $u_{j}, A_{j} \in C([0, \tau): Y)(j \in V)$ be Laplace transformable functions. Suppose that there exists a real number $\omega \geq \max \left(0, a b s\left(\left|a_{j}\right|\right)\right)(0 \leq j \leq n-1)$ such 
that $\quad \tilde{u}_{j}(\lambda) \in A_{j} \tilde{u}(\lambda), \quad \lambda>\omega, \quad j \in N_{n}^{0} \backslash V$ and $\overline{u_{j}, A_{j}}(\lambda)$ $2 A_{j}\left(\tilde{a}_{j}(\lambda) \tilde{u}(\lambda)\right), \lambda>\omega, j \in V$. Then an application of Lemma 2.2 yields that $u_{j}(t) \in A_{j} u(t), t \geq 0, j \in N_{n}^{0} \backslash V$ and $u_{j}, A_{j}(t) \in A_{j}\left(a_{j}^{*} u\right)(t), t \geq 0, j \in V$. If, in addition to this, there exists a continuous section $f \in \sec _{c}(F)$ that is Laplace transformable (not necessarily exponentially bounded) and:

$$
\sum_{j \in \mathbb{N}_{n}^{0} \backslash V} \tilde{a}_{j}(\lambda) \tilde{u}_{j}(\lambda)+\sum_{j \in V} \overline{u_{j}, A_{j}}(\lambda)+\tilde{f}(\lambda)=0, \lambda>\omega
$$

Then the uniqueness theorem for Laplace transform implies that $u(t)$ is a $V$-solution of problem (1.1).

Using the argumentation contained in the proofs of [(Bazhlekova, 2001), Theorem 3.1], [(Prüss, 1993), Theorem 4.3] and [(Kostić, 0000c), Theorem 4.8Theorem 4.9], we can simply deduce the following subordination principles for $\mathrm{V}$-solutions of problem (1.1); here it is worth noting that we can formulate a similar result provided that the multivalued linear operators employed in our analysis are relatively closed (Knuckles and Neubrander, 1994; Kostić, 0000c).

\section{Theorem 3.4}

Suppose that $\tau=\infty, \gamma \in(0,1), \omega>0,\left|a_{j}\right|(t)$ and $\left|b_{j}\right|(t)$ $(0 \leq j \leq n-1)$ satisfy $(\mathrm{P} 1), b_{n}(t):=\delta$-distribution and:

$$
\tilde{a}_{j}\left(\lambda^{\gamma}\right):=\tilde{b}_{j}(\lambda), \lambda>\omega
$$

Suppose that the MLOs $A_{j}$ are closed for $0 \leq j \leq n$. Let $u \in L T_{o r}-X$ be a $V$-solution of problem (1.1), with $F=$ $f \in L T_{o r}-Y$ being single-valued and the functions $u_{j} \in C([0$, $\tau): Y)\left(j \in N_{n}^{0} \backslash V\right), u_{j}, A_{j} \in C([0, \tau): Y)(j \in V)$, appearing in (3.1), being Laplace transformable and exponentially bounded. Define:

$$
\begin{aligned}
& u_{\gamma}(t):=\int_{0}^{\infty} t^{-\gamma} \Phi_{\gamma}\left(s t^{-\gamma}\right) u(s) d s, t>0, u_{\gamma}(0):=u(0), \\
& u_{j, \gamma}(t):=\int_{0}^{\infty} t^{-\gamma} \Phi_{\gamma}\left(s t^{-\gamma}\right) u_{j}(s) d s, \\
& t>0, u_{j, \gamma}(0):=u_{j}(0), j \in N_{n}^{0} \backslash V \\
& u_{j} A_{j, \gamma}(t):=\int_{0}^{\infty} t^{-\gamma} \Phi_{\gamma}\left(s t^{-\gamma}\right) u_{j}, A_{j}(s) d s, \\
& t>0, u_{j}, A_{j, \gamma}(0):=u_{j}, A_{j}(0), j \in V, \\
& f_{\gamma}(t):=\int_{0}^{\infty} t^{-\gamma} \Phi_{\gamma}\left(s t^{-\gamma}\right) f(s) d s, t>0, f_{\gamma}(0):=f(0)
\end{aligned}
$$

Then the function $u_{\gamma}(t)$ is an exponentially bounded $V$-solution of problem (1.1), with the functions $a_{j}(t)$ replaced by $b_{j}(t)(0 \leq j \leq n-1)$ and the in homogeneity $F(t)$ replaced by $f_{\gamma}(t)$. Furthermore, the functions $u_{j, \gamma} \in C([0$, $\tau): Y)\left(j \in N_{n}^{0} \backslash V\right)$ and $u_{j}, A_{j, \gamma} \in C([0, \tau): Y)(j \in V)$ satisfy the requirements from Definition 3.1(ii).

\section{Theorem 3.5}

Let $\left|a_{j}\right|(t),\left|b_{j}\right|(t)$ and $c(t)$ satisfy (P1), let $\beta \geq 0$ be such that $\int_{0}^{\infty} e^{-\beta t}\left|d_{j}(t)\right| d t<\infty, j \in N_{n-1}^{0}$ and let $A_{j}$ be closed for $0 \leq j \leq n, b_{n}(t):=\delta$-distribution Set:

$$
\alpha:=\tilde{c}^{-1}\left(\frac{1}{\beta}\right) \text { if } \int_{0}^{\infty} c(t) d t>\frac{1}{\beta}, \alpha:=0 \text { otherwise }
$$

Let $u^{b} \in L T_{o r}-X$ be a $V$-solution of problem (1.1), with the function $F=f^{b} \in L T_{o r}-Y$ being single-valued and let the set $\left\{e^{-\omega b t} u_{b}(t): t \geq 0\right\}$ be bounded in $X\left(\omega_{b} \geq 0\right)$. Let $u_{j}^{b} \in L T_{o r}-Y\left(j \in N_{n}^{0} \backslash V\right)$ and $u_{j}^{b}, A_{j} \in L T_{o r}-Y(j \in V)$ be the functions fulfilling the requirements from Definition 3.1(ii). Suppose that $c(t)$ is completely positive and:

$$
\tilde{b}_{j}(1 / \tilde{c}(\lambda))=\tilde{a}_{j}(\lambda), j \in N_{n-1}^{0}, \lambda>\alpha
$$

Let:

$$
\omega_{a}=\tilde{c}^{-1}\left(\frac{1}{\omega_{b}}\right) \text { if } \int_{0}^{\infty} c(t) d t>\frac{1}{\omega_{b}}, \omega_{a}=0 \text { otherwise }
$$

Then, for every $r \in(0,1]$, there exist locally Holder continuous (with the exponent $r \in(0,1])$ functions $u^{a, r} \in L T_{o r}-X, \quad u_{j}^{a, r} \in L T_{o r}-Y\left(j \in N_{n}^{0} \backslash V\right), \quad u_{j}^{a, r}, A_{j} \in L T_{o r}-Y$ $(j \in V)$ and $f^{\mathfrak{l} \in L T_{o r}}-Y$ such that:

$$
\begin{aligned}
& \widetilde{u^{a, r}}(\lambda)=\frac{1}{\pi^{1+r} \tilde{c}} \widetilde{u^{b}}(1 / \tilde{c}(\lambda)), \lambda>\alpha \\
& \widetilde{u_{j}^{a, r}}(\lambda)=\frac{1}{\lambda^{1+r} \tilde{c}(\lambda)} \widetilde{u_{j}^{b}}(1 / \tilde{c}(\lambda)), j \in \mathbb{N}_{n}^{0} \backslash V, \lambda>\alpha \\
& \widetilde{u_{j, A_{j}}^{a, r}}(\lambda)=\frac{1}{\lambda^{1+r} \tilde{c}(\lambda)} \widetilde{u_{j, A_{j}}^{b}}(1 / \tilde{c}(\lambda)), j \in \mathbb{N}_{n}^{0} \backslash V, \lambda>\alpha
\end{aligned}
$$

And:

$$
\widetilde{f^{a, r}}(\lambda)=\frac{1}{\lambda^{1+r} \tilde{c}(\lambda)} \widetilde{f^{b}}(1 / \tilde{c}(\lambda)) \text { for } \lambda>\alpha
$$

Furthermore, for every $r \in(0,1], u^{a, r}(t)$ is a $V$-solution of problem (1.1), with the function $F=f^{a, r}$, as well as the functions $u_{j}^{a, r}(t)\left(j \in N_{n}^{0} \backslash V\right)$ and $u_{j}^{a, r}, A_{j}(t) \quad(j \in V)$ satisfy the requirements from Definition 3.1(ii) and the set $\left\{e^{-}\right.$ $\left.\omega^{\omega a t} u^{a, r}(t): t \geq 0\right\}$ is bounded in $X$, if $\omega_{b}=0 \omega_{b} \tilde{c}(0) \neq 1$, resp., the set $\left\{e^{-\omega t} u^{a, r}(t): t \geq 0\right\}$ is bounded in $X$ for any $\in>0$, if $\omega_{b}>0$ and $\omega_{b} \tilde{c}(0)=1$. 
It is worth noting that the mapping $t \mapsto u_{\gamma}(t), t>0$ (cf. Theorem 3.4) admits an extension to $\sum_{\min \left(\left(\frac{1}{\gamma}-1\right) \frac{\pi}{2}, \pi\right)}$ and the mapping $\quad z \mapsto u_{\gamma}(z), z \in \sum_{\min \left(\left(\frac{1}{\gamma}-1\right) \frac{\pi}{2}, \pi\right)}$ is analytic. Furthermore, the solution $u_{\gamma}(t)$ possesses some other properties clarified in [(Kostić, 0000c), Theorem 4.8].

In the remaining part of this section, it will be assumed that $Z$ is a sequentially complete locally convex space. We would like to propose the following general definition.

\section{Definition 3.6}

Suppose that $k \in C([0, \tau)), \quad C_{1} \in L(Z, \quad Y) \quad$ and

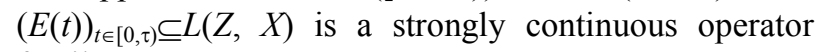
family.

It is said that $(E(t))_{t \in[0, \tau)}$ is a pre- $\left(k, C_{1}, V\right)$-existence family for (1.1) iff for each $z \in Z$ there exist continuous sections $\quad u_{j, z} \in \sec _{c}\left(A_{j} E(\cdot) z\right) \quad\left(j \in N_{n}^{0} \backslash V\right)$ such that $\left(a_{j}^{*} E(\cdot) z\right)(t) \in D\left(A_{j}\right), t \in[0, \tau), j \in V, z \in Z$ and:

$$
\begin{aligned}
& 0 \in \sum_{j \in N_{n}^{0} \backslash V}\left(a_{j} * u_{j, z}\right)(t)+\sum_{j \in V} A_{j}\left(a_{j} * E(\cdot) z\right)(t) \\
& +k(t) C_{1} z, t \in[0, \tau), z \in Z
\end{aligned}
$$

It is said that $(E(t))_{t \in[0, \tau)}$ is a $\left(k, C_{1}, V\right)$-existence family for (1.1) iff for each $z \in Z$ there exist continuous sections $\quad u_{j, z} \in \sec _{c}\left(A_{\mathrm{j}} E(\cdot) z\right) \quad\left(j \in N_{n}^{0} \backslash V\right)$ and $\quad u_{j}$, $A_{j, z} \in \sec _{c}\left(A\left(a_{j}^{*} E(\cdot) z\right)\right)$ such that:

$$
\begin{aligned}
& \sum_{j \in N_{n}^{0} \backslash V}\left(a_{j} * u_{j, z}\right)(t)+\sum_{j \in V} u_{j}, A_{j, z}(t) \\
& +k(t) C_{1} z,=0, t \in[0, \tau), z \in Z
\end{aligned}
$$

One can simply verify that the notion of a pre- $\left(k, C_{1}\right.$, $V$ )-existence family for (1.1) generalizes the notion of a $k$-regularized $C_{1}$-existence family introduced in [(Kostić, 2015b), Definition 3.8(i)], as well as the notion of a mild $(a, k)$-regularized $C_{1}$-existence family sub generated by a multivalued linear operator, introduced in [(Kostić, 0000c), Definition 3.8(i)]. It is clear that any $k$ regularized $C_{1}$-existence family, whose construction has been verified by applying [(Kostić, 2015b), Theorem $3.9(\mathrm{i}) /(\mathrm{b})]$, can serve as a particular example of a $\left(k, C_{1}\right.$, $V)$-existence family for (1.1), with the operators $A_{0}, \cdots, A_{n-}$ $1, B$ being singe-valued and the set $V$ appropriately chosen. Furthermore, if card $(V) \leq 1$, then any pre- $\left(k, C_{1}\right.$, $V)$-existence family for (1.1) is automatically a $\left(\mathrm{k}, C_{1}\right.$, $V)$-existence family for (1.1).

If $(E(t))_{t \in[0, \tau)}$ is a (pre-) $\left(k, C_{1}, V\right)$-existence family for (1.1), then it is clear that, for every $z \in Z$, the mapping
$u_{\mathrm{z}}(t):=E(t) z, t \in[0, \tau)$ is a $V($-pre)-solution of problem (1.1) with $F(t):=k(t) C_{1} z, t \in[0, \tau)$.

In our analyses of uniqueness of solutions of problem (1.1), it will be crucial for us to assume that $X=Y$. We will use the following definition, in which we generalize the notions introduced in [(Kostić, 2015b), Definition 3.8(ii)] and [(Kostić, 0000c), Definition 5.1(iii)].

\section{Definition 3.7}

Suppose that $k \in C([0, \tau)), C_{2} \in L(X)$ is injective,

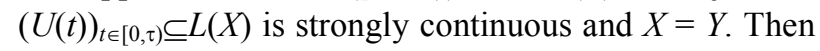
we say that $(U(t))_{t \in[0, \tau)}$ is a k-regularized $C_{2}$-uniqueness family for (1.1) iff:

$$
U(t) x_{n}+\sum_{j=0}^{n-1}\left(a_{j} * U(\cdot) x_{j}\right)+k(t) C_{2} x=0, t \in[0, \tau)
$$

whenever $x \in \cap_{j=0}^{n} D\left(A_{j}\right)$ and $x_{j} \in A_{j} x(0 \leq j \leq n)$.

Repeating almost literally the arguments given in the proof of [(Kostić, 2015a), Theorem 2.10.17(ii)], we can simply deduce the following proposition.

\section{Proposition 3.8}

Suppose that $k \in C([0, \tau)), k(t)$ is a kernel on $[0, \tau)$, $C_{2} \in L(X)$ is injective, $(U(t))_{t \in[0, \tau)}$ is a locally equicontinuous $k$-regularized $C_{2}$-uniqueness family for (1.1), $X=Y$ and the function $F=f:[0, \tau) \rightarrow Y$ is single-valued. Then the problem (1.1) has at most one strong solution.

Observe that, in the formulation of Proposition 3.8, we do not require the closedness of multivalued linear operators $A_{0}, \cdots, A_{n-1}, B$. Using Proposition 3.8, we can simply prove that there exists a unique $V$-solution of problem (1.1), provided the closedness of all operators $A_{0}, \cdots, A_{n-1}, \mathrm{~B}$ and the validity of conditions from Proposition 3.8 .

The class of exponentially equicontinuous $k$ regularized $C_{2}$-uniqueness families for (1.1) can be simply characterized by using the vector-valued Laplace transform.

\section{Theorem 3.9}

Let $k(t)$ and $\left|a_{j}\right|(t)(0 \leq j \leq n-1)$ satisfy (P1). Suppose that $\tau=\infty, k \in C([0, \infty)), C_{2} \in L(X)$ is injective, $(U(t)) t \geq 0$ is strongly continuous and the operator family $\left\{e^{-\omega t} U(t)\right.$ : $t \geq 0\} \subseteq L(X)$ is equicontinuous for some real number $\omega \geq \max \left(0, a b s(k), a b s\left(\left|a_{j}\right|\right)\right)(0 \leq j \leq n-1)$. Then $(U(t)) t \geq 0$ is a $k$-regularized $C_{2}$-uniqueness family for (1.1) iff the following holds:

$$
\int_{0}^{\infty} e^{-\lambda t} U(t)\left[x_{n}+\sum_{j=0}^{n-1} \tilde{a}_{j}(\lambda) x_{j}\right] d t=-\tilde{k}(\lambda) C_{2} x, \Re \lambda>\omega
$$

whenever $x \in \cap_{j=0}^{n} D\left(A_{j}\right)$ and $x_{\mathrm{j}} \in A_{j} x(0 \leq j \leq n)$. 
Now we can clarify the following subordination principle for k-regularized $C_{2}$-uniqueness families (cf. also [(Kostić, 2015b), Theorem 3.7(ii)]).

\section{Theorem 3.10}

Suppose that $\tau=\infty, k, k_{1} \in C([0, \in)), C_{2} \in L(X)$ is injective, $c(t)$ is completely positive, $c(t), k(t), k_{1}(t)$, $\left|\mathrm{a}_{0}\right|(t), \cdots, \quad\left|a_{n-1}\right|(t), \quad\left|\mathrm{b}_{0}\right|(t), \cdots, \quad\left|\mathrm{b}_{\mathrm{n}-1}\right|(t) \quad$ satisfy $(\mathrm{P} 1)$ and $(U(t))_{t \geq 0}$ is an exponentially equicontinuous $k$-regularized $C_{2}$-uniqueness family for problem (1.1), with the functions $a_{j}(t)$ replaced by functions $b_{j}(t)$ therein $(0 \leq j \leq n)$. Let $\omega_{0}>0$ be such that, for every $\lambda>\omega_{0}$ with $\tilde{c}(\lambda) \neq 0$ and $\tilde{k} 1 \backslash(\tilde{c}(\lambda)) \neq 0$, (3.4) holds. Then for each $r \in(0,1]$ there exists a locally Holder continuous (with exponent $r$ ), exponentially equicontinuous $\left(k_{1}{ }^{*} g_{r}\right)$ regularized $C_{2}$-uniqueness family for (1.1).

On the other hand, the class of exponentially equicontinuous pre- $\left(k, C_{1}, V\right)$-existence families for (1.1) cannot be so simply characterized with the help of vector-valued Laplace transform. As the next theorem shows, this is not the case with the class of exponentially equicontinuous $\left(k, C_{1}, V\right)$-existence families for (1.1); cf. also [(Kostić, 2015b), Theorem 3.9(i)/(a)].

\section{Theorem 3.11}

Suppose that $\tau=\infty, k \in C([0, \infty)), k(t)$ and $\left.\right|_{\mathrm{aj}} \mid(t)$ $(0 \leq j \leq n-1)$ satisfy $(\mathrm{P} 1)$ and $(E(t))_{t \geq 0}$ is a $\left(k, C_{1}, V\right)$ existence family for (1.1). Let $A_{j}$ be closed for $0 \leq j \leq n$. Suppose that there exists a real number $\omega \geq \max (0$, $\left.a b s(k), a b s\left(\left|a_{j}\right|\right)\right)(0 \leq j \leq n-1)$ such that the operator family $\left\{e^{-\omega t} E(t): \mathrm{t} \geq 0\right\} \subseteq L(Z, X)$ is equicontinuous, as well as that the continuous sections $u_{j, z} \in \sec _{c} \quad\left(A_{j} E(\cdot) z\right)$ $\left(j \in N_{n}^{0} \backslash V\right)$ and $u_{j}, \mathrm{~A}_{\mathrm{j}, z} \in \sec _{c}\left(A_{j}\left(a_{j}^{*} E(\cdot) z\right)\right)$, appearing in (3.5), are exponentially bounded with the exponential growth bound less than or equal to $\omega$ (and the meaning clear) Set:

$$
P_{\lambda}:=\sum_{j=0}^{n} \widetilde{a_{j}}(\lambda) A_{j}, \Re \lambda>\omega
$$

Then, for every $\lambda \in C$ with $\Re \lambda>\omega$ and $\tilde{a}_{j}(\lambda) \neq 0(j \in V)$, we have:

$$
\tilde{k}(\lambda) C_{1} z \in P_{\lambda} \tilde{E}(\lambda) z, z \in Z
$$

\section{Proof}

Performing the Laplace transform, we get:

$$
\begin{aligned}
& \sum_{j \in N_{n}^{0} \backslash V} \widetilde{a_{j}}(\lambda) \widetilde{u_{j, z}}(\lambda)+\sum_{j \in V} \widetilde{u_{j}, A_{j, z}}(\lambda)+\tilde{k}(\lambda) C_{1} z=0, \\
& \Re \lambda>\omega, z \in Z
\end{aligned}
$$

Since the operators $A_{j}$ are closed for $0 \leq j \leq n$, we can apply Lemma 2.1 in order to see that:

$$
\widetilde{u_{j, z}}(\lambda) \in A_{j} \tilde{E}(\lambda) z\left(j \in N_{n}^{0} \backslash V, \Re \lambda>\omega\right)
$$

And:

$$
\begin{aligned}
& \widetilde{u_{j, z} A_{j, z}}(\lambda) \in A_{j}\left(\widetilde{a_{j}^{*} E(\cdot) z}\right)(\lambda) \\
& =A_{j}\left[\widetilde{a_{j}}(\lambda) \tilde{E}(\lambda) z\right](j \in V, \Re \lambda>\omega)
\end{aligned}
$$

Substituting this in (3.6) and using the fact that $A_{j}\left[\widetilde{a_{j}}(\lambda) \tilde{E}(\lambda) z=\widetilde{a}_{j}(\lambda) A_{j} \tilde{E}(\lambda) z\right]$ $\left(j \in V, \Re \lambda>\omega, \widetilde{a}_{j}(\lambda) \neq 0\right)$, the result immediately follows.

The assertion of [(Kostić, 2015b), Theorem 3.9(i)/(b)] can be extended to multivalued linear operators by assuming some very restrictive additional conditions. Contrary to this, the assertion of [(Kostić, 2015b), Theorem 3.4(i)] admits a very simple reformulation in our context.

\section{Theorem 3.12}

Suppose that $0 \leq \alpha_{0}<\cdots<\alpha_{n}$ and $V=N_{n}^{0}$. Let $(E(t))_{t \in[0,}$, $\tau)$ be a $\left(g_{1}, \quad C_{1}, \quad V\right)$-existence family for (1.1), with $a_{j}(t):=g_{\alpha_{n}-\alpha j}(t)(0 \leq j \leq n-1)$ and let $0<T<\tau$. Suppose, futher, that $A_{j}$ is closed for $0 \leq j \leq n$. Set $m_{i}:=$ $\left\lceil\alpha_{\mathrm{i}}\right\rceil, i \in N_{n}^{0}$ and, for every $i \in N_{m_{n}-1}^{0}, D_{i}:=\left\{j \in N_{n-1}: m_{j^{-}}\right.$ $1 \geq i\}, D_{i}^{\prime}:=\left\{j \in N_{n-1}^{0}: m_{j}-1 \geq i\right\} \quad D_{i}^{\prime \prime}:=N_{n-1}^{0} \backslash D_{i}^{\prime}$ and:

$$
D_{i}:=\left\{u_{i} \in \bigcap_{j \in D_{i}^{\prime}} D\left(A_{j}\right): A_{j} u_{i} \cap R\left(C_{1}\right) \neq \phi, j \in D_{i}^{\prime \prime}\right\}
$$

If $u_{i} \in D_{i}$ for $0 \leq i \leq m_{n}-1$, then we define:

$u(t):=\sum_{i=0}^{m_{n}-1} u_{i} g_{i+1}(t)-\sum_{i=0}^{m_{n}-1} \sum_{j \in N_{n-1} \backslash D_{i}}\left(g_{\alpha_{n}-\alpha_{j}+i} * E\right)(t) v_{i, j}$

$-\sum_{i=0}^{m_{n}-1}\left(g_{\alpha_{n}-\alpha_{j}+i} * E\right)(t) v_{i, 0}, 0 \leq t \leq T$

where, $v_{i, j} \in Z$ satisfy $A_{j} u_{i} \cap C_{1} v_{\mathrm{i}, \mathrm{j}} \neq \phi$ for $0 \leq j \leq n-1$. Then the Caputo fractional derivative $D_{t}^{\zeta} u(t)$ is defined for any number $\zeta \in\left[0, \alpha_{n}\right]$ and, for every $j \in N_{n}^{0}$, there exists a continuous section $u_{j}, \alpha_{j}(t)$ of $A_{j} D_{t}^{\alpha_{j}}(t)$ on $[0, \tau)$ such that:

$$
\sum_{j=0}^{n} u_{j}, \alpha_{j}(t)=0,0 \leq t \leq T
$$


Before we briey describe how we can provide some illustrative examples of results obtained so far, it is worth noting that the assertion of [(Kostić, 2015b), Theorem 3.6], concerning the inhomogeneous problem (1.1) with single-valued linear operators $A_{j}=A_{j}(0 \leq j \leq n)$, cannot be extended to the multivalued linear operator case.

In [(Favini and Yagi, 1998), Example 6.1], the authors have considered a damped Poisson-wave equation in the space $X:=L^{2}(\Omega)$, where $\Omega \subseteq R^{n}$ is a bounded open domain with smooth boundary. Let $m(x) \in L^{\infty}(\Omega), m(x) \geq 0$ a.e. $x \in \Omega$, let $\Delta$ be the Dirichlet Laplacian in $L^{2}(\Omega)$, acting with domain $H_{1}^{0}(\Omega) \cap H^{2}(\Omega)$ and let $A(x ; D)$ be a second order linear differential operator on with coefficients continuous on $\bar{\Omega}$. Using the analysis from the above-mentioned example (cf. also the proof of [(Favini and Yagi, 1998), Theorem 6.1]), it readily follows from Theorem 3.9 that there exists $\zeta>0$ such that there exists an exponentially bounded $g_{\zeta}$ regularized $\Delta(1-\Delta)^{-1}$-uniqueness family for the problem (1.1), with $n=2, B=B \in L(X)$ being the scalar multiplication with $m(x), \mathrm{A}_{0}:=-\Delta, A_{1}:=A(x ; D):=A$, $a_{0}(t):=g_{1}(t)$ and $a_{1}(t):=g_{2}(t)$. Applying Theorem 3.5 and Theorem 3.10, we obtain that for a large class of inhomogeneities $f(t, x)$ there exists a unique solution of the abstract degenerate Volterra integral equation:

$$
\begin{aligned}
& m(x) u(t, x)-\int_{0}^{t} c(t-s) \Delta u(s, x) d s \\
& +A \int_{0}^{t}(c * c)(t-s) u(s, x) d s=f(t, x), t \geq 0, x \in \Omega
\end{aligned}
$$

where, $c(t)$ is a completely positive function. Observe, finally, that the subordination principles clarified in Theorem 3.4-Theorem 3.5 and Theorem 3.10 can be applied to many other problems from [(Favini and Yagi, 1998), Chapter VI], as well.

Suppose that $\mathrm{A}$ is a closed sub generator of an exponentially equicontinuous $\left(g_{\delta}, g_{\sigma}\right)$-regularized Cresolvent family $\left(R_{\delta}(t)\right)_{t \geq 0}$, in the sense of [(Kostić, 0000c), Definition 5.2] $(0<\delta \leq 2, \sigma \geq 1)$. Then the analyses contained in [(Kostić, 2015a), Example 2.10.30(i)] and Remark 3.2, along with Theorem 3.9, can be used for proving some results on the existence and uniqueness of solutions of the abstract degenerate Volterra inclusion:

$$
0 \in u(t)+\sum_{j=1}^{n-1} c_{j}\left(g_{\alpha_{n}-\alpha_{j}} * u\right)(t)-A\left(g_{\alpha_{n}} * u\right)(t)+f(t), t \geq 0
$$

where, $0 \leq \alpha_{1}<\alpha_{2}<\cdots<\alpha_{n}<\delta$ and $c_{j} \in C(1 \leq j \leq n-1)$.

Consider the equation:

$$
\begin{aligned}
& (v-\Delta) u_{t}=\Delta u+\int_{0}^{t} g(t-s) \Delta u(s, x) d s, t>0, x \in \Omega ; \\
& u(0, x)=\phi(x), x \in \Omega
\end{aligned}
$$

In $L^{2}(\Omega)$, where $\phi \neq \Omega \subseteq R^{n}$ is a bounded domain with smooth boundary $\partial \Omega, g \in \mathrm{L}^{1}{ }_{l o c}([0, \infty)), \Delta$ denotes the Dirichlet Laplacian and $v=\lambda_{k 0}$ is an eigenvalue of $\Delta$; cf. also Example 4.4 below. Using the theory of integrated existence and uniqueness families, we have proved in [(Kostić, 0000a), Example 2.2.43(iii)] that for each function $\phi \in H^{2}(\Omega) \cap H^{1}(\Omega)$ there exists a unique strong solution $t \mapsto u(t), t \geq 0$ of the associated once integrated integral equation:

$$
(v-\Delta) u(t)=t(v-\Delta) \phi(x)+\left(g_{1} * g * \Delta u\right), t \geq 0
$$

Furthermore, the mappings $t \mapsto u(t), t>0$ and $t \mapsto u(t), t>0$ can be analytically extended to the sector $\sum_{\pi / 2}$.

\section{On analytical Solutions of Abstract Degenerate Multi-Term Fractional Problems with Caputo Derivatives}

In this section, we investigate the existence and uniqueness of analytical solutions of abstract degenerate Cauchy problem (1.2), provided that there exists an index $i \in N_{n}^{0}$ such that $\alpha_{i} \notin N_{0}$, for the sake of brevity, we consider only the homogeneous case. Let us recall that $n \in M\{1\}, A, B$ and $A_{1}, \cdots, A_{n-1}$ are closed linear operators on a sequentially complete locally convex space $X, 0 \leq \alpha_{1}$ $<\cdots<\alpha_{n}, 0 \leq \alpha<\alpha_{n}$ and $D_{t}^{\alpha}$ denotes the Caputo fractional derivative of order $\alpha$. Define $A_{n}:=B, A_{0}:=\mathrm{A}, m:=\lceil\alpha\rceil$, $\alpha_{0}:=\alpha, m_{i}:=\left\lceil\alpha_{i}\right\rceil, \quad i \in N_{n}^{0}$ and $D_{i}:=\left\{j \in N_{n-1}: m_{j}-1\right.$ $\geq i\}\left(i \in N_{m_{n}-1}^{0}\right)$.

By a strong solution of (1.2), we mean any function $u \in C^{m_{n}-1}([0, \infty): X)$ such that the Caputo fractional derivative $D_{t}^{\alpha_{n}} u(t)$ is well-defined, as well as that the terms $B D_{t}^{\alpha_{n}} u(t), \quad A_{i} D_{t}^{\alpha_{i}} u(t)(1 \leq i \leq n-1)$ and $A D_{t}^{\alpha} u(t)$ are well-defined and continuous for $t \geq 0$ and that (1.2) holds identically for $t \geq 0$.

We will use the following definition of an analytical solution of problem (1.2).

\section{Definition 4.1}

A function $u \in C([0, \infty): X)$ is said to be an analytical solution of problem (1.2) on the region $\mathrm{C} \backslash(-\infty, 0]$ iff $u(\cdot)$ is a strong solution of (1.2) and it can be extended to the whole complex plane, analytically on the region $\mathrm{C} \backslash(-\infty$, $0]$ and continuously on the region $C \backslash(-\infty, 0)$, as well as any of the terms $A_{i} D_{t}^{\alpha_{i}} u(t)(0 \leq i \leq n, \mathrm{t} \geq 0)$ is well defined and can be extended to the whole complex plane, 
analytically on the region $C \backslash(-\infty, 0]$ and continuously on the region $\mathrm{C} \backslash(-\infty, 0)$ :

Set, for every $\gamma \in(-\pi, \pi]$ and $z \in C \backslash\{0\}$ :

$$
\begin{aligned}
& P_{z, \gamma}:=B+\sum_{j=1}^{n-1} z^{\alpha_{j}-\alpha_{n}} e^{i \gamma}\left(\alpha_{n}-\alpha_{j}\right) \\
& A_{j}-z^{\alpha-\alpha_{n}} e^{i \gamma}\left(\alpha_{n}-\alpha\right) A ; P_{z}:=P_{z, 0}
\end{aligned}
$$

Before stating the following theorem, it should be observed that $P_{z, \gamma}$ need not be equal to $P_{z e-i \gamma}$ for some particular values of $\gamma$ and $z$ :

\section{Theorem 4.2}

Suppose $k(t)$ satisfies (P1), $C_{1} \in L(Y, X), C_{2} \in L(X)$ is injective and there exists a locally equicontinuous $k$ regularized $C_{2}$-uniqueness family for problem (1.2), with $f(t) \equiv 0$ (in the sense of [(Kostić, 2015b), Definition 3.11]). Suppose that for each number $\gamma \in(-\pi, \pi]$ there exists a sufficiently large number $R>0$ such that the operator $P_{z, \gamma}$ is injective for $|z| \geq R$, as well as that the operator families $\left\{P_{z, \gamma}^{-1} C_{1}:|z| \geq R, z \notin[-\infty, 0)\right\} \subseteq L(Y, X)$ and $\left\{z^{\alpha_{j}-\alpha_{n}} A_{j} P_{z, \gamma}^{-1} C_{1}:|z| \geq R, j \in N_{n}^{0}, z \notin[-\infty, 0)\right\} \subseteq L(Y, X) \quad$ are equicontinuous. Let the mappings $z \mapsto P_{z, \gamma}^{-1} C_{1} y \in X,|z|$ $\notin(-\infty, 0]$ and $P_{z, \gamma}^{-1} C_{1} y=D y,|z|>R, z \notin(-\infty, 0]$ be analytic for any $y \in Y, j \in N_{n}^{0}, \gamma \in(-\pi, \pi]$ and let there exist operators $D, \quad D_{0}, \quad D_{1}, \cdots, D_{n} \in L(Y, X)$ such that $\lim _{\mathrm{z} \rightarrow+\infty} P_{z, \gamma}^{-1} C_{1} y=D y$ and $\lim _{z \rightarrow+\infty} z^{\alpha_{j}-\alpha_{n}} e^{i_{y}\left(\alpha_{n}, \alpha_{j}\right)} A_{j} P_{z}^{-1} C_{1} y=$ $D_{j} y$ for any $y \in Y, j \in \mathbb{N}_{n}^{0} \quad \gamma \in(-\pi, \pi]$. Suppose that $u_{i} \in D_{i}$ for $0 \leq i \leq m_{n}-1$. Then there exists a unique analytical solution of problem (1.2) on the region $\mathbb{C} \backslash(-\infty, 0]$, with $f(t) \equiv 0$. Denote by $A_{i} D_{z}^{\alpha_{i}} u(z)$ the analytical extensions of terms $A_{i} D_{z}^{\alpha_{i}} u(z)(0 \leq i \leq n, t \geq 0)$ on region $\mathbb{C} \backslash(-\infty, 0]$. Then, for every $\zeta \in(0, \pi)$, we have the existence of a positive real number $\omega^{\prime}>0$ such that the sets $\left\{e^{-\omega^{\prime} z} u(z): z \in \overline{\Sigma_{\zeta}}\right\}$ and $\left\{e^{-\omega^{\prime} z} A_{i} D_{z}^{\alpha_{i}} u(z): z \in \overline{\Sigma_{\zeta}}\right\}(0 \leq i \leq n)$ are bounded in $\mathrm{X}$.

\section{Proof}

The proof of theorem is very similar to that of [(Kostić, 2016), Theorem 2.2] and, because of that, we will only outline the most relevant details.

Fix numbers $\gamma \in(-\pi, \pi]$ and $\zeta \in(0, \pi)$. Then, for every $\theta \in(0, \pi / 2)$, there exists a sufficiently large number $\omega_{\gamma, \theta}>$ 0 satisfying that the function $r_{\gamma, \theta} \quad(z)$ := $z^{-1} P_{z, \gamma}^{-1} C_{1} \in L(Y, X), \quad z \in \omega_{\gamma, \theta}+\sum_{\theta+(\pi / 2)} \quad$ is $\quad$ well-defined, strongly analytic and that for each $\theta^{\prime} \in(0, \theta)$ the operator family $\left\{z^{-1}\left(z-\omega_{\gamma, \theta}\right) \quad P_{z, \gamma}^{-1} C_{1}: z \in \omega_{\gamma, \theta}+\Sigma_{\theta^{\prime}+(\pi / 2)}\right\} \subseteq L(Y, X)$ is equicontinuous. Arguing as in the proof of [(Kostić, 2016), Theorem 2.2], we obtain the existence of a strongly analytic operator family $\left(W_{\gamma}(z)\right)_{z \in \Sigma_{\pi / 2} \subseteq} L(Y, X)$ satisfying that $\lim _{z \rightarrow 0, z \in \Sigma_{\theta}} W_{\gamma}(z) y=D y(\theta \in(0, \pi / 2), y \in Y)$, $\int_{0}^{\infty} e^{-z t} W_{\gamma}(t) y d t=z^{-1} P_{z, \gamma}^{-1} C_{1} y, \Re z>\omega_{\gamma, \theta}, y \in Y$ and that, for every $\theta \in(0, \pi / 2),\left\{e^{-\omega_{\gamma, \theta^{z}}} W_{\gamma}(z): z \in \Sigma_{\theta^{\prime}}\right\} \subseteq L(Y, X)$ is an equicontinuous operator family $\left(\theta^{\prime} \in(0, \theta)\right)$.

Define:

$$
W(z):=\left\{\begin{array}{l}
W_{0}(z), z \in \Sigma_{\pi / 2}, \\
W_{\pi / 2}\left(z e^{-i \pi / 2}\right), \text { if } z \in e^{i \pi / 2} \Sigma_{\pi / 2}, \\
W_{\theta \pi / 2}\left(z e^{i \pi / 2}\right), \text { if } z \in e^{-i \pi / 2} \Sigma_{\pi / 2} \\
0, \text { if } z<0, \\
D, \text { if } z=0 .
\end{array}\right.
$$

Then it can be simply verified that $W(\cdot)$ is well-defined as well as that there exists $\omega>0$ such that the operator family $\left\{e^{-\omega z} W(z): z \in \overline{\Sigma_{\zeta}}\right\} \subseteq L(Y, X)$ is equicontinuous as well as that, for every $y \in Y$, the mapping $z \mapsto W(z) y, z \in \mathbb{C} \backslash(-\infty, 0]$ is analytic. Replacing the function $z \mapsto q_{\gamma, \theta}(z)$, $z \in \omega_{\gamma, \theta}+\Sigma_{\theta+(\pi / 2)}$ with the function $z \mapsto q_{\gamma, \theta, j}(z):=$ $z^{-1} z^{\alpha_{j}-\alpha_{n}} e^{i \gamma\left(\alpha_{n}-\alpha_{j}\right)} \quad A_{j} P_{z, \gamma}^{-1} C_{1} \in L(Y, X), z \in \omega_{\gamma, \theta}+\Sigma_{\theta+(\pi / 2)}$ in the first part of proof $\left(\theta \in(0, \pi / 2), j \in \mathbb{N}_{n}^{0}\right)$, we can define a strongly analytic operator family $\left(W_{\gamma, j}(z)\right)_{z \in \Sigma_{\pi / 2}} \subseteq L(Y, X)$ satisfying that, for every $\theta \in(0, \pi / 2)$ and $y \in Y$, we have that $\int_{0}^{\infty} e^{-z t} W_{\gamma, j}(t) y d t=z^{-1} z^{\alpha_{j}-\alpha_{n}} e^{i \gamma\left(\alpha_{n}-\alpha_{j}\right)} A_{j} P_{z, \gamma}^{-1} C_{1} y, \Re z>\omega_{\beta, \theta}$ and the operator family $\left\{e^{-\omega_{\beta, \theta^{z}}} W_{\gamma, j}(z): z \in \Sigma \theta^{\prime}\right\} \subseteq L(Y, X)$ is equicontinuous $\left(\theta \in(0, \theta), j \in \mathbb{N}_{n}^{0}\right)$.

Define now $W^{j}(\cdot)$ by replacing $W_{0}(\cdot), W_{\pi / 2}(\cdot), W_{\text {. }}$ $\pi / 2(\cdot)$ and $D$ in the definition of $W(\cdot)$ with $W_{0, j}(\cdot)$; $W_{\pi / 2, j}(\cdot), W_{-\pi / 2, j}(\cdot)$ and $D_{j}$, respectively $\left(j \in \mathbb{N}_{n}^{0}\right)$. Then there exists $\omega_{j}>0$ such that the operator family $\left\{e^{-\omega_{j} z} W^{j}(z): z \in \overline{\Sigma_{\zeta}}\right\} \subseteq L(Y, X)$ is equicontinuous as well as that, for every $y \in Y$, the mapping $z \mapsto W^{j}(z) y$, $z \in \mathbb{C} \backslash(-\infty, 0]$ is analytic and the mapping $z \mapsto W^{j}(z) y$, $z \in \mathbb{C} \backslash(-\infty, 0)$ is continuous $\left(j \in \mathbb{N}_{n}^{0}\right)$.

By [(Kostić, 2015b), Theorem 3.5(i)/(b)], we get that there exists an exponentially equicontinuous $C_{1}$-existence family for (1.2), denoted by $(E(t))_{t \geq 0}$ (in the sense of [(Kostić, 2015b), Definition 3.1(i)]; cf. also Definition 3.6 for a slightly generalized notion). Furthermore, for every 
$y \in Y$, the mapping $t \mapsto E(t) y, t \geq 0$ can be analytically extended to the region $z \in \mathbb{C} \backslash(-\infty, 0]$ so that the mapping $z \mapsto E(z) y, \quad z \in \mathbb{C} \backslash(-\infty, 0)$ is continuous as well as that $E^{\left(m_{n}-1\right)}(z) y=W(z) y, \quad z \in \mathbb{C} \backslash(-\infty, \quad 0], \quad y \in Y \quad$ and $A_{j}\left(g_{\alpha_{n}-\alpha_{j}} * E^{\left(m_{n}-1\right)}\right)(z) y=W^{j}(z) y, z \in \mathbb{C} \backslash(-\infty, 0], y \in Y, j \in \mathbb{N}_{n}^{0}$ (this equality can be proved by using the closedness of operators $A_{j}$ for $j \in \mathbb{N}_{n}^{0}$, Lemma 2.2 and the arguments already used in the proofs of Kato's analyticity criteria from [(Kostić, 2011), Section 2.4]). By [(Kostić, 2015b), Theorem 3.4(i)], we get that the function $t \mapsto u(t), t \geq 0$, given by (3.7), with $E(\cdot)$ replaced therein with $E^{\left(m_{n}-1\right)}(\cdot)$, is a unique strong solution of problem (1.2).

The remaining part of proof can be obtained by using a slight modification of the corresponding parts of the proof of [(Kostić, 2016), Theorem 2.2].

\section{Remark 4.3}

Suppose that $Y=X, C_{1} \in L(X)$ is injective, $C_{1} A_{j} \subseteq$ $A_{j} C_{1}, j \in \mathbb{N}_{n}^{0}$, as well as that the operator families $\left\{P_{z, \gamma}^{-1}\right.$

$\left.C_{1}:|z| \geq R, z \notin(-\infty, 0]\right\} \subseteq L(X)$ and $z^{\alpha_{j}-\alpha_{n}} A_{j} P_{z, \gamma}^{-1} C_{1}:|z| \geq R$, $\left.z \notin \quad(-\infty, 0], j \in \mathbb{N}_{n}^{0}\right\} \subseteq L(X)$ are equicontinuous and strongly continuous for any $\gamma \in(-\pi, \pi]$. Then the analyticity of mappings $z \mapsto P_{z, \gamma}^{-1} C_{1} x \in X,|z|>R, z \notin(-\infty$, 0] and $z \mapsto z^{\alpha_{j}-\alpha_{n}} A_{j} P_{z, \gamma}^{-1} C_{1} x \in X,|z|>R, z \notin(-\infty, 0]$ automatically follows for any $x \in X, j \in \mathbb{N}_{n}^{0}, \gamma \in(-\pi, \pi]$ (cf. the proof of [(Sviridyuk and Zamyshlyaeva, 2006), Theorem 1.1] and apply the identity $(1+z)^{\zeta}=$ $\left.\sum_{k=0}^{\infty}\left(\begin{array}{l}\zeta \\ k\end{array}\right) z^{k}|z|<1, \zeta>0\right)$.

Now we will analyze fractional analogons of the abstract fractional Boussinesq-Love equation and the abstract Barenblatt-Zheltov-Kochina equation in finite domains.

\section{Example 4.4}

Suppose that $\emptyset \neq \Omega \subseteq \mathbb{R}^{n}$ is a bounded domain with smooth boundary $\partial \Omega$. Denote by $\left\{\lambda_{k}\right\}$ the eigenvalues of the Dirichlet Laplacian $\Delta$ in $L^{2}(\Omega)$ (recall that $0<-\lambda_{1} \leq$ $\lambda_{2} \ldots \leq-\lambda_{\mathrm{k}} \leq \ldots \rightarrow+\infty$ as $\left.k \rightarrow \infty\right)$ numbered in nonascending order with regard to multiplicities; by $\left\{\phi_{k}\right\} \subseteq C^{\infty}(\Omega)$ we denote the corresponding set of mutually orthogonal [in the sense of $L^{2}(\Omega)$ ] eigenfunctions.

Let $0<\alpha_{1}<\alpha_{2} \leq 2$. We consider the following fractional degenerate Cauchy-Dirichlet problem:

$$
\begin{aligned}
& (\lambda-\Delta) D_{t}^{\alpha_{2}}(t, x)-\alpha\left(\Delta-\lambda^{\prime}\right) D_{t}^{\alpha_{1}}(t, x) \\
& =\beta\left(\Delta-\lambda^{\prime \prime}\right) u(t, x), t \geq 0, x \in \Omega
\end{aligned}
$$

$$
\begin{aligned}
& u(0, x)=u_{0}(x) ; u_{t}(0, x) \\
& =u_{1}(x),(t, x) \in[0, \infty) \times \Omega \text { if } \alpha_{2}>1 \\
& u(t, x)=0,(t, x) \in[0, \infty) \times \partial \Omega
\end{aligned}
$$

where, $\Delta$ denotes the Dirichlet Laplacian on $L^{2}(\Omega), \lambda, \lambda^{\prime}$, $\lambda^{\prime \prime} \in \mathbb{R}, \alpha, \beta \in \mathbb{R}$ and $\beta \neq 0$. Assume that the following condition holds:

$$
\lambda \in \sigma(\Delta) \wedge \lambda \neq \lambda^{\prime} \wedge\left(\alpha=0 \Rightarrow \lambda \neq \lambda^{\prime \prime}\right)
$$

Arguing similarly as in Example following [(Kostić, 2016), Remark 2.3], we can prove that the requirements of Theorem 4.2 holds with $D=D_{0}=D_{1}$ $=D_{2}=0$, so that the abstract Cauchy problem (4.1)(4.3) has a unique analytical solution on the region $\mathbb{C} \backslash(-\infty, \quad 0]$ for any $u_{0}(x) \in H^{2}(\Omega) \cap H_{0}^{1}(\Omega)$ and $u_{1}(x) \in H^{2}(\Omega) \cap H_{0}^{1}(\Omega)$, if $\alpha_{2}>1$.

Let $\alpha \in(0,2) \backslash\{1\}$, let $X=Y=L^{2}(\Omega)$ and let $\Delta$ be the Dirichlet Laplacian on $X$. Using Parseval's equality, it can be easily seen that the operator $D: \mathrm{f} \mapsto(-1)(\zeta \lambda)^{-1}$ $\sum_{\lambda=\lambda_{k}}\left\langle\phi_{k}, f\right\rangle\left(f \in L^{2}(\Omega)\right)$ belongs to the space $L\left(L^{2}(\Omega)\right)$ : Consider the following fractional Barenblatt-ZheltovKochina equation:

$(\lambda-\Delta) D_{t}^{\alpha}(t, x)=\zeta \Delta u(t, x), t \in \mathbb{R}, x \in \Omega$

$u(0, x)=u_{0}(x), x \in \Omega ; u_{t}(0, x)$

$=u_{1}(x),(t, x) \in[0, \infty) \times \Omega$, if $\alpha>1$

$u(t, x)=0,(t, x) \in \mathbb{R} \times \partial \Omega$

where, $\zeta \in \mathbb{R} \backslash\{0\}$ and $\lambda=\lambda_{k_{0}} \in \sigma(\Delta)$ (observe that the problem (4.4)-(4.6) is a special case of problem (1.2) with $n=2, B=\lambda-\Delta, A_{1}=0, A=\zeta \Delta, \alpha_{2}=\alpha$ and $\alpha_{1}=\alpha=$ 0 , clearly, $\left.P_{z}=\lambda-\left(1+\zeta z^{-\alpha}\right) \Delta\right)$. Let $\gamma \in(-\pi, \pi]$. Then it is not difficult to verify that, for every $\theta \in(0, \pi / 2)$, there exists a sufficiently large number $\omega_{\gamma, \theta}>0$ satisfying that the function $q_{\gamma, \theta}(z):=z^{-\alpha-1} P_{z, \gamma}^{-1} \in L(X), z \in \omega_{\gamma, \theta}+\Sigma_{\theta+(\pi / 2)}$ is well-defined, strongly analytic and that for each $\theta^{\prime} \in(0, \theta)$ the operator family $\left\{z^{-\alpha-1} P_{z, \gamma}^{-1}: z \in \omega_{\gamma, \theta}+\Sigma_{\theta^{\prime}+(\pi / 2)}\right\} \subseteq L(X), z$ is equicontinuous. Furthermore, for every $f \in X$, there exists an $X$-valued analytic mapping $z \mapsto W_{\gamma, f}^{\alpha}(z), z \in \Sigma_{\pi / 2}$ satisfying that, for every $\theta \in(0, \pi / 2)$, one has $\int_{0}^{\infty} e^{-z t} W_{\lambda, f}^{\alpha}(t) d t=z^{-\alpha-1} P_{z, \gamma}^{-1} f, \Re z>\omega_{\gamma, \theta}$ and the set $\left\{e^{-\omega \gamma, \theta^{z}} W_{\gamma, f}^{\alpha}(z): z \in \Sigma_{\theta^{\prime}}\right\}$ is bounded in $X\left(f \in X, \theta^{\prime} \in(0, \theta)\right)$. Define $W_{\gamma}^{\alpha}(z) f:=W_{\gamma, f}^{\alpha}(z), z \in \Sigma_{\pi / 2}, f \in X$. Then, for every 
$\theta \in \quad(0, \quad \pi / 2), \quad\left\{e^{-\omega \gamma, \theta^{z}} W_{\gamma}^{\alpha}(z): z \in \Sigma_{\theta}\right\} \in L(X) \quad$ is $\quad$ an equicontinuous operator family. By the foregoing, we have $z^{-\alpha} P_{z}^{-1} f \rightarrow D f$, as $|z| \rightarrow \infty, z \notin(-\infty, 0](f \in X)$, similarly, the operator family $\left\{z^{-\alpha-1} B P_{z}^{-1}:|z| \geq R, z \notin(-\right.$ $\infty 1,0]\} \in L(X)$ is equicontinuous and $z^{-\alpha} B P_{z}^{-1} f \rightarrow 0$, $|z| \rightarrow 1, z \notin(-\infty, 0](f \in X)$, so that we can define a strongly analytic operator family $\left(W_{\gamma, B}^{\alpha}(z)\right)_{z \in \Sigma \pi / 2} \subseteq L(X)$ satisfying that, for every $\theta \in(0, \pi / 2)$, the operator family $\left\{e^{-\omega_{, \theta}^{\prime} z} W_{\beta, B}^{\alpha}(z): z \in \Sigma_{\theta}\right\} \subseteq L(X)$ is equicontinuous for some number $\omega_{\gamma, \theta}^{\prime}>0$. We have that, for every $f \in X$ and $\theta \in(0$, $\pi / 2), \lim _{z \rightarrow 0, z \in \Sigma_{\theta}} W_{\gamma}^{\alpha}(z) f=D f$. Define:

$$
W^{\alpha}(z):=\left\{\begin{array}{l}
W_{0}^{\alpha}(z), z \in \Sigma_{\pi / 2}, \\
e^{i \alpha \pi / 2} W_{\pi / 2}^{\alpha}\left(z e^{-i \pi / 2}\right), \text { if } z \in e^{i \pi / 2} \Sigma_{\pi / 2}, \\
e^{-i \alpha \pi / 2} W_{-\pi / 2}^{\alpha}\left(z e^{-i \pi / 2}\right), \text { if } z \in e^{-i \pi / 2} \Sigma_{\pi / 2} \\
0, \text { if } z<0 \\
D, \text { if } z=0
\end{array}\right.
$$

and $W_{B}^{\alpha}(z)$ by replacing the operators $W_{0}^{\alpha}(z), W_{\pi / 2}^{\alpha}\left(z e^{-}\right.$ $i \pi / 2$ ).

$W_{-\pi / 2}^{\alpha}\left(z e^{i \pi / 2}\right)$ and $D$ in the above definition by the operators $W_{0, B}^{\alpha}(z), W_{\pi / 2, B}^{\alpha}\left(z e^{-i \pi / 2}\right), W_{-\pi / 2, B}^{\alpha}\left(z e^{i \pi / 2}\right)$ and 0 , respectively $(z \in \mathbb{C})$. Then, for every $v \in(0, \pi)$, there exists a finite constant $\omega_{v}>0$ such that the operator families $\quad\left\{e^{-\omega_{v} z} W^{\alpha}(z): z \in \overline{\Sigma_{v}}\right\} \subseteq \quad L(X) \quad$ and $\left\{e^{-\omega_{v} z} B W^{\alpha}(z): z \in \overline{\Sigma_{v}}\right\}=\left\{e^{-\omega z} W^{\alpha, B}(z): z \in \overline{\Sigma_{v}}\right\} \subseteq L(X)$ are equicontinuous as well as that, for every $f \in X$, the mappings $z \mapsto W^{\alpha}(z) f$ and $z \mapsto B W^{\alpha}(z) f$ are analytical on $\mathbb{C} \backslash(-\infty, 0]$ and continuous on $\mathbb{C} \backslash(-\infty, 0)$. Now it can be easily seen that $\left(W^{\alpha}(t)\right)_{t \geq 0} \subseteq L(X,[D(B)])$ is an exponentially bounded $\left(g_{\alpha}, g_{\alpha+1}\right)$-regularized resolvent family generated by $A, B$, in the sense of [(Kostić, 0000b), Definition 2.5]. Furthermore, there exists a sufficiently large number $R^{\prime}>0$ such that $(z B-A)^{-1} \in L(X)$ for all $z \in S_{R^{\prime}}:=\left\{z \in \mathbb{C} \backslash(-\infty, 0]:|z|=R^{\prime}\right\}$. Denote, for every $f \in H^{2}(\Omega) \cap H_{0}^{1}(\Omega)$ and $z \in S_{R^{\prime}}$ :

$$
\begin{aligned}
& u(t ; f, z):=-W^{\alpha}(t) B f \\
& -\int_{0}^{t}(t-s)^{\alpha-1} E_{\alpha, \alpha}\left((t-s)^{\alpha-1} z\right) W^{\alpha}(s) x d s \\
& +E_{\alpha}\left(t^{\alpha} z\right)(z B-A)^{-1} B f, t \geq 0
\end{aligned}
$$

Fix a number $z_{0} \in S_{R^{\prime}}$ such that $z_{0} \mathrm{e}^{ \pm i \alpha / 2} \in S_{R^{\prime}}$. Using [(Kostić, 0000b), Theorem 2.6], it readily follows that the function $t \mapsto u\left(t, u_{0}, z_{0}\right)+\int_{0}^{t} u\left(s, u_{1}, z_{0}\right) d s, t \geq 0$ is a unique solution of problem (4.4)-(4.6) with the initial values $\mathrm{u} 0$ and $\mathrm{u} 1$ replaced by $\left(z_{0} B-A\right)^{-1} B u_{0}$ and $\left(z_{0} B-A\right)^{-1}$ $B u_{1}$, respectively. On the other hand, an elementary argumentation concerning analytical Rproperties of vector-valued Laplace transform shows that the function $t \mapsto \int_{0}^{t}(t-s)^{\alpha-1} E_{\alpha, \alpha}\left((t-s)^{\alpha-1} z\right) W^{\alpha}(s) x d s, t \geq 0$ can be continuously extended on the region $\mathbb{C} \backslash(-\infty, 0)$, analytically on the region $\mathbb{C} \backslash(-\infty, 0]$, by the formula:

$$
\left\{\begin{array}{l}
L^{-1}\left(\frac{1}{z^{\alpha}-z_{0}} \frac{1}{z^{\alpha+1}}\right), \text { if } z \in \Sigma_{\pi / 2}, \\
L^{-1}\left(\frac{1}{z^{\alpha}-e^{i \pi \alpha / 2} z_{0}} \frac{1}{z^{\alpha}+1} P_{z, \pi / 2}^{-1}\right), \text { if } z \in e^{i \pi / 2} \Sigma_{\pi / 2}, \\
L^{-1}\left(\frac{1}{z^{\alpha}-e^{-i \pi \alpha / 2} z_{0}} \frac{1}{z^{\alpha}+1} P_{z,-\pi / 2}^{-1}\right), \text { if } z \in e^{-i \pi / 2} \Sigma_{\pi / 2}, \\
0, \text { if } z \leq 0 .
\end{array}\right.
$$

This simply implies that, for any two functions $u_{0}, u_{1}$ $\in H^{2}(\Omega) \cap H_{0}^{1}(\Omega)$, orthogonal to the eigenfunction(s) $\phi_{k}$ for $k=k_{0}$, there exists a unique analytical solution $t$ $\mapsto u(t), t \geq 0$ of problem (4.4)-(4.6) on the region $\mathbb{C} \backslash(-\infty$, $0]$ with the property that for each $v \in(0, \pi)$ there exists a finite constant $\omega_{v}>0$ such that the sets $\left\{e^{-\omega_{v} z} u(t): z \in \overline{\Sigma_{v}}\right\}$ and $\left\{e^{-\omega_{v} z} B u(t): z \in \overline{\Sigma_{v}}\right\}$ are bounded.

\section{Conclusion}

The theory of abstract degenerate Volterra integrodifferential equations is still very undeveloped. We deeply believe that this paper will motivate our researchers to continue the work within this field of mathematics.

\section{Acknowledgment}

The author would like to express his sincere thanks to the anonymous referee for the careful reading of paper.

\section{Ethics}

The author hereby declares no conflict of interest with regard to this manuscript.

\section{References}

Arendt, W., C.J.K. Batty, M. Hieber and F. Neubrander, 2001. Vector-valued Laplace Transforms and Cauchy Problems. 1st Edn., Springer Science and Business Media, Basel, ISBN-10: 3764365498, pp: 523.

Bazhlekova, E., 2001. Fractional evolution equations in Banach spaces. Ph.D. Thesis, Eindhoven University of Technology, Eindhoven. 
Carroll, R.W. and R.W. Showalter, 1976. Singular and Degenerate Cauchy Problems. 1st Edn., Academic Press, New York, ISBN-10: 0121614506, pp: 333.

Cross, R., 1998. Multivalued Linear Operators. 1st Edn., CRC Press, ISBN-10: 0824702190, pp: 352.

Demidenko, G.V. and S.V. Uspenskii, 2003. Partial Differential Equations and Systems not Solvable With Respect to the Highest-Order Derivative. 1st Edn., CRC Press, ISBN-10: 0824748514, pp: 632.

Diethelm, K., 2010. The Analysis of Fractional Differential Equations. 1st Edn., Springer, ISBN-10: 3642145752, pp: 264.

Favini, A. and A. Yagi, 1998. Degenerate Differential Equations in Banach Spaces. 1st Edn., CRC Press, ISBN-10: 0824716779, pp: 336.

Favini, A. and H. Tanabe, 2001. Degenerate volterra equations in banach spaces. Differ. Integral Equ., 14: 613-640.

Fedorov, V.E. and A. Debbouche, 2013. A class of degenerate fractional evolution systems in Banach spaces. Differ. Equ., 49: 1569-1576. DOI: $10.1134 / \mathrm{S} 0012266113120112$

Kilbas, A.A.A., H.M. Srivastava and J.J. Trujillo, 2006. Theory and Applications of Fractional Differential Equations. 1st Edn., Elsevier, Amsterdam, ISBN-10: 0444518320, pp: 523.

Kim, M., 2002. Volterra inclusions in Banach spaces. Rocky Mountain J. Math., 32: 167-178. DOI: $10.1216 / \mathrm{rmjm} / 1030539615$

Knuckles, C. and F. Neubrander, 1994. Remarks on the Cauchy Problem for Multi-Valued Linear Operators. In: Partial Differential Equations, Akademie-Verlag, Berlin, pp: 174-187.

Kostić, M., 2011. Generalized Semigroups and Cosine Functions. 1st Edn., Mathematical Institute SANU, Belgrade, ISBN-10: 8680593451, pp: 352.

Kostić, M., 2015a. Abstract Volterra Integro-Differential Equations. 1st Edn., CRC Press, Boca Raton, ISBN-10: 148225431X, pp: 484.

Kostić, M. 2015b. Degenerate k-regularized $\left(\mathrm{C}_{1}, \mathrm{C}_{2}\right)$ existence and uniqueness families, CUBO, 17: 15-41. DOI: 10.4067/S0719-06462015000300002

Kostić, M., 0000a. Abstract degenerate volterra integrodifferential equations: Linear theory and applications. Book Manuscript.
Kostić, M., 0000b. Degenerate multi-term fractional differential equations in locally convex spaces. Publ. Inst. Math., Nouv. Ser.

Kostić, M., 0000c. Abstract degenerate Volterra inclusions in locally convex spaces. Preprint

Kostić, M., 2016. On entire solutions of abstract degenerate differential equations of higher order. Funct. Anal. Approx. Comput., 8: 51-60.

Lizama, C., 2000. Regularized solutions for abstract Volterra equations. J. Math. Anal. Applic., 243: 278-292. DOI: 10.1006/jmaa.1999.6668

Martinez, C. and M. Sanz, 2001. The Theory of Fractional Powers of Operators. 1st Edn., Elsevier, ISBN-10: 0080519075, pp: 378.

Melnikova, I.V. and A.I. Filinkov, 2001. Abstract Cauchy Problems: Three Approaches. 1st Edn., Chapman and Hall/CRC, Boca Raton, ISBN-10: 1420035495, pp: 264.

Obukhovskii, V. and P. Zecca, 2003. On boundary value problems for degenerate differential inclusions in Banach spaces. Abstr. Applied Anal., 13: 769-784. DOI: $10.1155 / \mathrm{S} 108533750330301 \mathrm{X}$

Podlubny, I., 1999. Fractional Differential Equations. 1st Edn., Academic Press, New York, ISBN-10: 0125588402, pp: 340.

Prüss, J., 1993. Evolutionary Integral Equations and Applications. 1st Edn., Birkhèauser Verlag, Basel, ISBN-10: 0817628762, pp: 366.

Samko, S.G., A.A. Kilbas and O.I. Marichev, 1993. Fractional Integrals and Derivatives: Theory and Applications. 1st Edn., Gordon and Breach, Yverdon, ISBN-10: 2881248640, pp: 1016.

Sviridyuk, G.A. and A.A. Zamyshlyaeva, 2006. The phase spaces of a class of linear higher-order Sobolev type equations. Differ. Equ., 42: 269-278. DOI: $10.1134 / \mathrm{S} 0012266106020145$

Sviridyuk, G.A. and V.E. Fedorov, 2003. Linear Sobolev Type Equations and Degenerate Semigroups of Operators. 1st Edn., Walter de Gruyter, ISBN-10: 3110915502, pp: 224.

Xiao, T.J. and J. Liang, 1998. The Cauchy Problem for Higher Order Abstract Differential Equations. 1st Edn., Springer Science and Business Media, New York, ISBN-10: 3540652388, pp: 300. 